\title{
Evaluating Capacitive Touch Input on Clothes
}

\author{
Paul Holleis \\ Albrecht Schmidt \\ University of Duisburg-Essen \\ Schützenbahn 70, 45117 Essen \\ paul@hcilab.org \\ albrecht.schmidt@acm.org
}

\author{
Susanna Paasovaara \\ Arto Puikkonen \\ Nokia Research Center Tampere \\ Visiokatu 1. 33720 Tampere \\ susanna.paasovaara@nokia.com \\ arto.puikkonen@nokia.com
}

\author{
Jonna Häkkilä \\ Nokia Research Center Oulu \\ Yrttipellontie 6, 90230 Oulu \\ jonna.hakkila@nokia.com
}

\begin{abstract}
Wearable computing and smart clothing have attracted a lot of attention in the last years. For a variety of applications, it can be seen as potential future direction of mobile user interfaces. In this paper, we concentrate on usability and applicability issues concerned with capacitive touch input on clothing. To be able to perform user studies, we built a generic platform for attaching, e.g., capacitive sensors of different types. On top of that, several prototypes of wearable accessories and clothing and implemented various application scenarios. We report on two studies we undertook with these implementations with a user group randomly sampled at a shopping mall. We provide a significant set of guidelines and lessons learned that emerged from our experiences and those studies. Thus, developers of similar projects have to put major efforts into minimizing the delay between button activation and feedback and to make location and identification of controls and their function as simple and quick as possible. Issues that have to be treated in all designs include the requirement of onehanded interaction and that, even for minimal functionality, to find a general solution with regard to layout and button-tofunction mapping is hardly possible. Additionally, in order to generate a satisfactory user experience good usability must be combined with aesthetical factors.
\end{abstract}

\section{Categories and Subject Descriptors}

H.5.2 User Interfaces: Haptic I/O, Prototyping

H.1.2 User/Machine Systems: Human factors

B.4.2 Input/Output Devices

\section{General Terms}

Human Factors, Experimentation, Design

\section{Keywords}

Capacitive touch, wearable controls, input on textiles, design guidelines

Permission to make digital or hard copies of all or part of this work for personal or classroom use is granted without fee provided that copies are not made or distributed for profit or commercial advantage and that copies bear this notice and the full citation on the first page. To copy otherwise, or republish, to post on servers or to redistribute to lists, requires prior specific permission and/or a fee.

MobileHCI 2008, September 2-5, 2008, Amsterdam, the Netherlands. Copyright (C) 2008 ACM 978-1-59593-952-4/08/09...\$5.00.

\section{INTRODUCTION}

Advances in computing technology have lead to computing devices in different forms to be present in our everyday life. Mobility is a phenomenon that has taken giant steps during the recent decade e.g. due to component miniaturization, improvements in energy consumption and developments in communication infrastructure. The use of mobile computing devices such as mobile phones, MP3 players, and PDAs have become a part of common practices, and overall omnipresence of the technology is gradually approaching Weiser's vision of Ubiquitous Computing [32] by becoming a truly integrated part of our society and personal life.

Despite of the high adoption rate with mobile computing, the input technologies have not yet evolved to an optimal level with regard to usability. Almost without exceptions, the manual control of a mobile device happens with buttons that are integrated into the gadget. This results in a large number of small buttons placed in a small physical area. Such input devices can sometimes be difficult to use especially if they are placed, e.g., in a pocket or handbag. To overcome the problem, remote controllers and headsets with input buttons have been introduced. However, these need to be remembered by the users to be taken along and their physical form factor significantly influences the comfort of carrying them. Additionally, if the control has to be held in one hand and controlled by the other, this leaves no hand free for other activities. Other suggested controls are integrated, e.g., in headphones or headphone cables. This means, however, that the location of the controls is defined by the device and not by usability aspects. Speech input technology has been developed to offer hands-free interactions, but besides accuracy issues, in practice the useful situations are often limited, e.g. due to social situations where silent use is often preferred.

Wearable computing offers an interesting approach for integrating new input methods to mobile computing technology and hence shows potential in mobile HCI. The term wearable computing generally refers to a small computer attached to its user in some way other than holding it. The main characteristics of wearable computers are that they are always accessible by the user and that the user can continue various activities while using them. Wearable computing offers large areas available for placing input controls and can embed controls into users' normal clothing.

Wearable computing can utilize smart textiles which constitute an underlying technology for wearable computers (e.g. power and data lines integrated into clothing). An ultimate goal of wearable computing is that all technology is completely and seamlessly integrated into clothing or wearable accessories like bags or 
today's glasses. The research on wearable computing has so far concentrated on demonstrating new concepts and applications. Systematic studies on the performance, preferences, expectations and acceptability of such technology have been rare. However, studying these aspects is important for creating usable and successful products, and can offer valuable insights for future designers of wearable computing applications.

As has been described by Rantanen et al. in [23], besides the technological and material aspects, a piece of intelligent clothing has also to provide the esthetical and functional properties expected from clothing in general.

In this paper we present different wearable prototypes controlling mobile technology that were developed to study the user experience with the technology. We present results of an extensive, two-phase user study concentrating on usability and acceptability issues with the technology, and discuss about general guidelines and lessons learned with designing wearable input technology.

\section{RELATED WORK}

The process of integrating electronics into textiles has been studied for some time now. It is still not yet entirely possible to fulfill the goal of having garments with all the characteristics of usual clothes as well as a network of sensors, input and output features, wireless connection and enough power to run for a satisfactory period of time without the need to recharge it. However, there have been enormous advances in textile fabrication processes as well as in the understanding of integrating conductive yarn.

[14] ("E-broidery") and [20] ("Electronic Textiles") give in-depth overviews about the subject of smart textiles. It covers early work like the Georgia Tech Wearable Motherboard [19], as well as current work like the wearable digital MP3 player [6] by Jung et al. The authors point to a number of challenges and visions as well as technological advances that drive the wearable computing community.

In the following, we deliberately leave out a more detailed treatment of the huge range of wearable devices that are either carried or in some other way obtrusively alter the way people wear clothes. We refer to, e.g., overviews like "Wearables in 2005" [24] and the review by Randell [22] for pointers to such projects and products, and mention those only if they have a distinct relation to our work.

Most research done in the field of smart clothing can be split into four categories. Much effort has gone into a technology driven approach that will enable systems and applications to be built. This includes research in the miniaturization of devices and power supplies, wireless communication methods as well as the integration of conductive wires into available clothing. Other researchers and product designers have focused on providing ideas and solutions for very specific areas of applications and usage settings. This includes specific environments as well as particular tasks. In recent years, the development of wearable systems and smart textiles has received increased attention. This includes actual hardware components as well as software frameworks that aid in the connection and interaction of such components. Only few results can be found about actual studies that consider the usability and the acceptance of such wearable controls in general, c.f. section 2.4 'Studies with Wearables'.

A general overview and collection of issues, end-user needs and appropriate technology has been assembled through several years of experience with designers, technologists and industrial collaborators by McCann et al. in [17].

The rest of this section treats each category in more detail and gives additional pointers to research projects.

\subsection{Technology Driven Projects}

A huge step towards the goal of including electronic components like sensors, actuators and computing platforms into clothing is achieved when these elements can be integrated into the sewing process. The second important quality of such components must be to seamlessly integrate into the comfort and original qualities of the garment. One way of encasing electronic circuits in a way that they are protected from power and data lines, do not depart from the design of the clothing and still enable the normal use like folding it is described in [4]. Button shaped casings are used to hide electronics, provide connections that can be sewn and preserve a cloth-like look as buttons often appear for fastening pockets or even for mere design purposes.

Linz, Kallmayer et al. show in [10] how to use flexible electronic modules and a way of connecting them with conductive yarn. The system allows using common fabrication processes and does not change the flexibility and feel of the textiles used. Although the requirements put on the yarn (can be sewn by a machine and must be conductive on the outside) place some restrictions on the material and their use, the reliability tests done by the authors show promising results. Metal wires are rather inflexible, however, and can reduce the wearablitity of the garment. Wade and Asada [30] describe a setup of a cable-free body area network which is based on conductive fabrics that are supposed to behave like normal fabrics. They provide a two wire bus that transmits power and data to sensor nodes. These nodes can potentially be located anywhere on a piece of garment and can take power from a single battery. Details about the two wire bus system can be found in [31]. Since one of the central issues in such conductive circuits is power loss because of the wiring, the placement of nodes will in practice be restricted. Wade and Asada set up a model to predict the resistance of large parts of a body shaped piece of textile. This can give hints as to where nodes should be placed most effectively.

A closer presentation and examination of different types of yarn that can be used for such purposes as well as their properties regarding knitting, yarn control, relaxation and geometry of knitted structures is found in [21].

These projects can be understood as enabling technology for creating smart textiles, especially for mass production scenarios. They can all be applied to several parts of the setups we used. [18] ('Fabric Computer Interfaces') shows what could already be done in 1998 with respect to fabric computing interfaces.

\subsection{Application Focused Projects}

There are many more projects for specific application areas that use some instance of wearable computing than can be recounted here. However, the vast majority of those uses larger additional devices like wrist keyboards or focus much more on the output or implicit input side. This includes a variety of head-up displays, 
headsets, watches or other body-worn displays well known for museum and tourist guide scenarios. Implicit input is realized by motion trackers and systems using health monitoring sensors. Explicit and direct input of users has mostly been studied for various methods of text input. One of the famous examples is the Twiddler system [13] which is a one-handed chording keyboard that has been demonstrated to outperform the multi-tap standard for mobile text entry. Other modern data input methods include speech recognition and data gloves that use several sensors to retrieve hand position and gestures (see, e.g., the glove of [11] which is used for text input). Both approaches have advantages but also, among others, the disadvantages that they often lack social acceptability, need heavy processing, are still unreliable and impose not negligible amounts of training on the user.

Focusing on the design for a specific application domain, Rantanen et al. [23] give an example of the design of a wearable interactive system for a particular type of environment. Their vest as well as their combined input and output device (appropriately called the YoYo interface), is designed especially for an arctic environment which requires, e.g., the use of gloves during the interaction.

A slightly different approach is taken by B. H. Thomas who demonstrates the e-SUIT in [27]. The paper shows how to augment a standard business-type suit with input and output features in a way to minimize the social weight, i.e. the "measure of the degradation of social interaction that occurs between the user and other people caused by the use of that item of technology". This means that the location of, e.g., buttons is not controlled by ease of finding, accessing or using them, but more from a social acceptability point of view. However, as stated in that paper, questions of look and feel, location, etc. are open research questions. The placement of the interaction items like buttons has been based on the author's judgment. We extend this work by presenting study-based results for the acceptance, location and type of controls to be used.

\subsection{Development Support}

To bring the development of wearable systems forward and to enable more people to implement their own ideas of smart textiles, it is vital to provide frameworks and toolkits. These can then be used to reduce the complexity of building such systems. In order to rapidly construct textiles using simple input, output and processing units, one can employ the recently published construction kit for electronic textiles described by Buechley in [1]. It offers temperature, light and pressure sensors, LEDs and small vibration motors, as well as a programmable microcontroller. The components are constructed in a way that helps connecting them to conductive wires. It is as such similar to the tools that we used ourselves to create the settings in our studies. The toolkit's rather bulky and standardized components do not seem to offer so easy customization possibilities of their appearance, however. The toolkit includes a pressure sensitive button but does not use any (e.g. capacitive) touch sensing components. Obviously, the foremost obstacle in creating a running application is still the programming of the microcontroller to process all the data and implement the logics. However, it won't take long until such assistive systems like the d.tools project [3] include hardware support for the special requirements of wearable computing. Other projects, e.g. [33], focus more on a model-driven approach and abstract descriptions to enable the semi-automatic generation of different user interfaces for display devices like PDAs or head-up displays.

\subsection{Studies with Wearables}

Some interesting projects have emerged that can assist those that want to develop or evaluate ideas and systems in the wearable domain. Knight et al. [8] present a methodology to evaluate wearable systems. Among other things they concentrate on physiological and biomechanical aspects as well as the comfort wearing or carrying such a system. The last aspect is analyzed in more detail by Knight and Baber in [7] where they also give tool support in assessing the comfort of large wearable computers.

More focusing on the development process, Hurford et al. [5] provide an interesting result from a survey that the user centered design approach is still only rarely taken in the field of wearable computing.

Of value for the paper at hand are also especially publications that concentrate on studying the location of controls on the human body. The well known analysis from Gemperle et al. [2] shows details about where on the human body solid and flexible forms can be attached best. This study focuses on places when the person is moving and finds places that are most stable during motions. The authors of [28] briefly describe a study to find out where people would want to place a small touch pad during different activities like sitting and standing. The results do not seem to be entirely conclusive but suggest, e.g., that the front of the thigh is the best place for placing such an input device. The main driving factors of the study to find optimal placements of optical input devices on the human body described in the technical report of Mayol et al [16] are the possible occlusion by the wearer, a clear sight to the main workspace of the user and movements of body parts that can influence the retrieved image. Since we do not use cameras for input and focus on public, every day use, aspects like reachability and social acceptability are more important in this context.

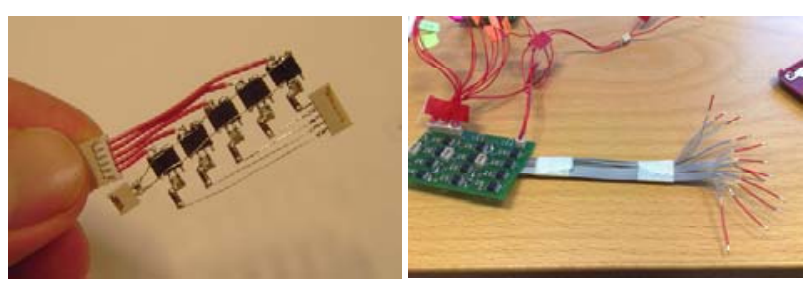

Figure 1: Two versions of assembled sensors ( 5 and 12 sensors with different connections). The size of the microcontroller and Bluetooth module is roughly the same.

\section{PROTOTYPES}

As has been described in the previous section, there are a few projects that attempt to theoretically describe users' views on different aspects on wearable computing. Since, however, there is still restricted knowledge of people about the possibilities and opportunities that wearable computing offers, we strongly argue in favor of giving people demonstrators and prototypes at hand. This can significantly increase the precision and validity of people's responses and is necessary to effectively find out about users' opinions, fears and acceptance of such ideas. We thus report on the set of prototypical devices and garment we built. These can be seen as an enabler for testing, demonstrating, and studying such applications. 


\subsection{Technology}

The four categories of prototypes developed for these studies all rely on the same type of technology although slightly different sensors and controller boards can be used for different packaging restrictions.

The underlying principle of the touch sensors we used is capacitive sensing. This technique has been in use for a long time already, one of the notable early uses being the musical instrument invented by Theremin in 1919. Since then it has already been used in touch sensitive tablets, e.g. [9] and nowadays the controls of modern stoves, washing machines and the like are often equipped with touch controls based on capacitive sensing. Without going into too much detail, the method relies on an excitation source that is connected to a transmitter generating an electric field to a receiver. The field lines are measured at the receiver by a $\Sigma-\Delta$ converter. When a grounded object interferes with the electric field, some of the field lines are shunted to ground and do not reach the receiver. Therefore, the total capacitance measured at the receiver can be used to detect the proximity or touch of an approaching object like a finger. In other words, capacitance changes according to the proximity of a finger and this change can be measured by a simple electronic setup.

Depending on the application setting, we use QT110 chips from Quantum Research that can each be used for one button or an Analog Devices AD7142 which supports up to 12 independent capacitance sensors. The latter has advantages when most sensitive areas are close together. It also has built-in algorithms for compensation for environmental changes and custom calibration registers. The single sensors are more appropriate if touch enabled areas are more distant from each other since the sensors can then be attached closer to the sensitive areas and it is less sensitive to touches close to the cables. Figure 1 shows two versions of the sensor part. Because the structure on the left does not use a circuit board, it is flexible and can also be used in parts of clothing that needs slight bending.

Using a Microchip PIC2550 microcontroller, the sensors are calibrated and all values are read at a frequency of about $25 \mathrm{~Hz}$. Whenever a change in the values is registered, an event is generated and sent through an attached Bluetooth module (Linkmatik 2.0).

This platform enables designers to quickly add touch controls to nearly arbitrary clothing, accessories and other devices. The only need is to integrate areas with conductive material (which is available as strings, sheets, etc. and attach them to the platform. The microcontroller and sensors can be powered using a variety of batteries ranging from about 3 to 12 Volt. Our prototypes either use an external small battery pack or are powered directly from a phone's battery. An overview of the system is shown in Figure 2.

Any device that supports the serial communication over Bluetooth protocol can then wirelessly connect to the sensor board. We implemented applications that react on touch input running on Series 60 phones, Nokia N800 internet tablets and PCs using J2ME and Python for Series 60, Python for Unix systems and Java/C++, respectively.

The whole system consisting of microcontroller, Bluetooth module, some additional required components, a few lamps for status display and the AD7142 sensor is not larger than a small mobile phone battery, about $2 \times 4 \times 1 \mathrm{~cm}$ in size.

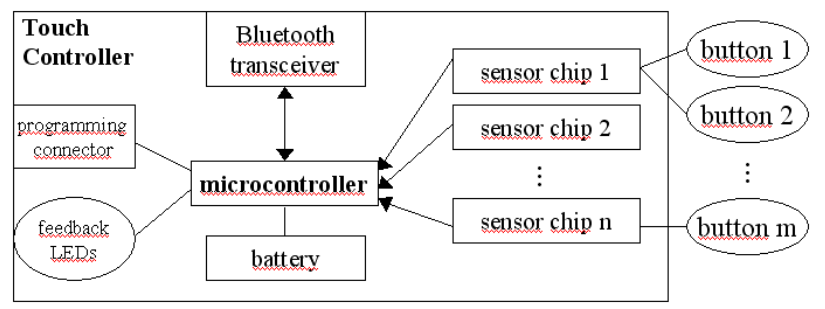

Figure 2: Overview of the architecture of the whole system.

The overall price of one such system is dominated by the Bluetooth module. The one we use costs about $110 €$ as a single item. There are much cheaper modules (this Linkmatik, e.g., supports an unnecessarily large range of up to 100 meters) which are, however, more difficult to use in a non-automated process. All other items (microcontroller, sensors, etc.) add up to approximately $10 €$. This price is only valid for developers who want to use one or several of those systems; the price tag will of course be significantly less with higher volumes.

\subsection{Phone Bags}

We created two sample versions of touch controls on off-the-shelf mobile phone bags, one is shown in Figure 3. The touch sensitive areas were made from thin conductive wires. The areas were intended to be integral parts of the existing design or shaped in ornamental forms themselves so as to constitute an interesting design that does not immediately suggest its button functionality.

Building on the fact that many people use their mobile as a music player, we added five buttons for the common controls to start/pause a song, skip forward, skip backward, turn the volume up, and turn it down.

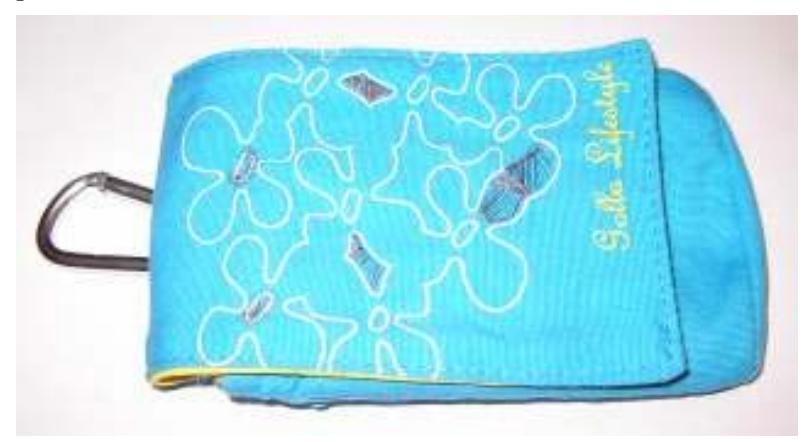

Figure 3: One of the phone bags with 5 touch areas (darker spots) integrated in the existing design.

The demonstration implementation was mainly targeted to control the music player function of a phone placed inside the bag. A J2ME application directly receives Bluetooth messages and controls the playback of available music files. Since all the application we built conform to some simple messaging standards, it is also possible to use it - without modifications of any sort - to control an N800 internet tablet or a home cinema application running on a PC (as the one we describe in Study II in section 4.2.2). 


\subsection{Helmet}

As a second example of a wearable accessory, a standard bicycle helmet has been equipped with two buttons realized as two larger touch sensitive areas. We undertook a quick set of trials to find out the optimal location and shape of these areas. It was chosen to be on the left and right side of the helmet such that it is easy to find them. This tackles the problem that one cannot see where the controls are while wearing the helmet. Since the touch controls do not need to completely cover the whole area, it was easy to integrate them into the existing design by using conductive foil to imitate the manufacturer's name and logo as well as some ornaments like lines or arrows, see Figure 4. The small controller and battery were attached to the helmet in a place where it should not alter the safety features of the helmet.

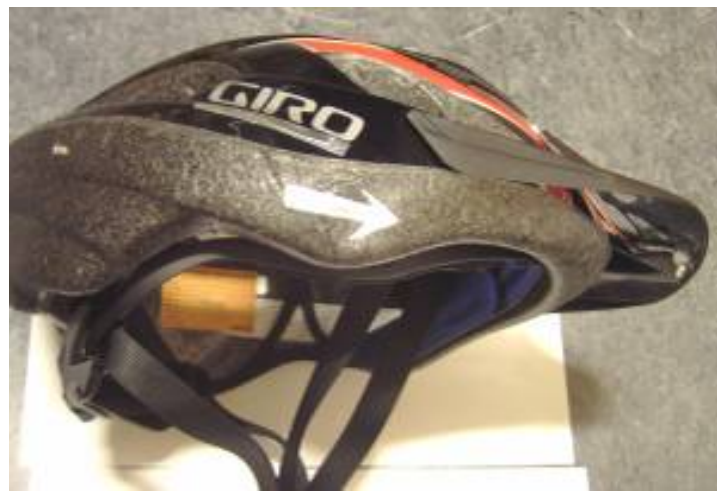

Figure 4: An off-the-shelf bicycle helmet that has been equipped with touch sensitive areas on both sides ('GIRO' logo and the arrow). A lamp in the front can give feedback.

\subsection{Gloves}

We built a versatile test implementation of gloves with controls on the back of the palm that can be controlled using the index finger of the other hand. The necessary modifications of the gloves are in fact very small and can be implemented very cheaply. As can be seen in Figure 5, the index finger of the control hand has been extended with a small patch of conductive yawn to enhance the capacity flow to the sensor. The more expensive electronic parts (e.g. Bluetooth module) are meant to be attached separately. Since especially working gloves are replaced in shorter intervals, this ensures that the additional cost is kept to a minimum. In the test prototype, different sets and layouts of controls can be attached on the glove using a small plug and Velcro tape. In a final version, this could of course also be embroidered into the glove.

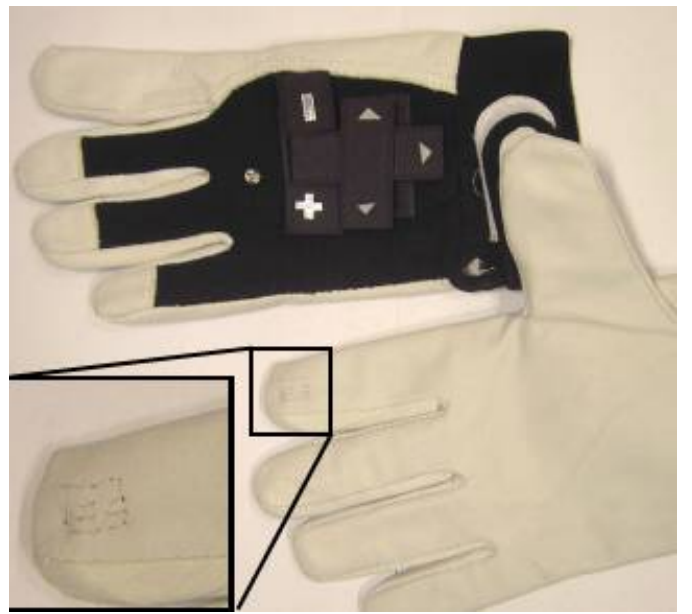

Figure 5: The prototypes used to demonstrate the idea of adding touch input on gloves. Some configurable buttons are placed on the left palm. The right index finger is used to initiate commands.

\subsection{Apron}

For a larger study of direct input on clothing, we built an apron equipped with three different implementations of a set of touch button (Figure 6) and a small pocket for the electronics and, possibly, a phone. One of the major issues in finding out about how and what people would want and accept, is the interplay between functionality and fashion. To find out more about this, we use 3 different button styles: visible button like shapes, buttons indistinguishable from ornaments, and nearly invisible buttons.

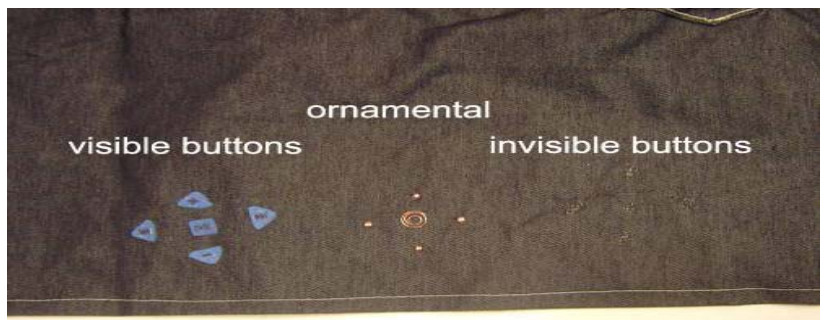

Figure 6: A close-up of the apron with the 3 different designs of the touch buttons.

In Figure 8, it can be seen how wearing the apron looks like. Sure enough, some male participants had the impression of wearing a skirt but got quickly used to it, especially since we placed some part of the studies in a kitchen scenario.

There are three obvious reasons for choosing an apron. First of all, it is a stand-alone piece of clothing that can be used, besides its use as a standard apron as a remote control. More importantly however, it served as a simulation tool for controls embedded into a skirt, trouser, or pair of shorts. Such an approach drastically eased the study process. Several versions can be tested relieving participants from having to change clothes in the beginning and during the course of a study. In contrast to other attachments, the apron still conveys the feeling that it is closely attached to the body, moves with the motions of the wearer's body and also otherwise behaves like a normal piece of garment. 
Second, this additionally enables the use of only one piece of technologically enhanced garment that fits all sizes and we can use one and the same sensor, processing and communication platform for different layouts and types of controls. And thirdly, it also fulfills a second important prerequisite of our study that people have the freedom of slightly adjusting and relocating the set of controls on the body. This possibility of quickly and unconsciously rearranging controls gives much better results about optimal positions than just having users try to imagine those locations.

\section{USER STUDIES}

In order to evaluate the wearable inputs, two user studies were arranged. Before each study, a pilot with one participant was held in order to refine the questions and to time the sessions to have an appropriate length.

\subsection{Participants}

The two user studies were done during July and August 2007 in Finland. The first study included 10 participants, and was arranged in a public shopping mall at a commercial city café table, where passers-by were recruited as test participants in an ad hoc fashion.

For the second user study, 8 participants were pre-recruited for a one hour test session each. In the beginning of the second study, the questions and tasks of the first study were repeated in a semipublic cafeteria environment increasing the number of participants for the first study to 18 . This was followed by the second part of the study, which was carried out in a usability laboratory.

In the tests, there were an equal number of male and female participants, with ages ranging from 16 to 30 years. Young people were chosen as a target group for the study as such types of technology typically enter the market first at this group. This appeared to be true as all of the test participants had mobile phones and 15 of the total 18 also owned MP3-players. To get an idea of how 'wearable' their current habits to use mobile technology was, we also asked where they stored their mobile devices at the time of the test. Only one participant was carrying an MP3 player at the time of the interview, but everyone had their mobile phone with them. All female participants carried their phone in a bag but half of them responded to have it sometimes also in their trouser pocket and one person in her jacket pocket. With regard to male participants, 7 of 9 had the phone in their trouser pocket correlating with their handedness, and 2 carried the phone mostly in their bag.

\subsection{Method and Tasks}

\subsubsection{Study I}

The first user study was held in a public place, a city café, and consisted of a semi-structured interview during which the participant could try out the prototypes, see Figure 7 . The gathered material was based on the direct answers to the questions as well as on observations. Three researchers were involved in the interviewing situation - one interacting with the user, and the other ones observing the users and preparing the prototypes. Each test session took approximately 20 minutes.
In the beginning, ten background questions were asked. After that, the bag, helmet and gloves were shown to the participant, in this order. With each prototype, the impressions and opinions of the participant about these were gathered. In the end, the participants were asked which of the ideas were their favorite and the reasons for that. Finally, they were asked about the benefits and obstacles they saw with wearable computing and to demonstrate where (on the body) they would like to place such input controls. To facilitate the question about placing the wearable input, we introduced the users with the idea of a near-toeye display with the possibility to play computer games or watch videos unnoticed by others, thus needing a suitable set of wearable controls.

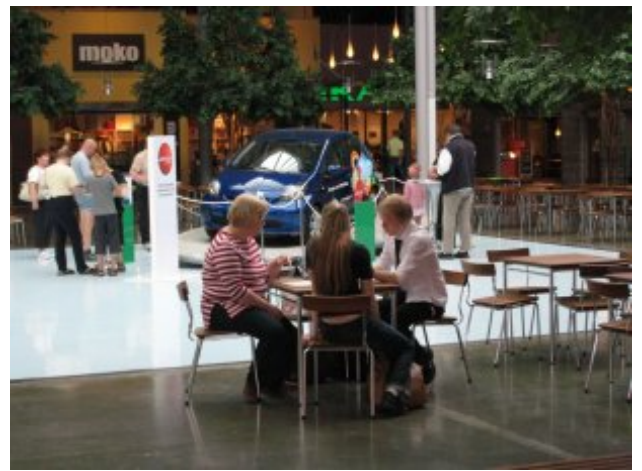

Figure 7: Study set-up in a public shopping mall for Study I.

\subsubsection{Study II}

The second study took approximately one hour per participant and consisted of two parts. The first part of Study II was held in a semi-public restaurant and had an identical setup with Study I.

The second part of the Study II was arranged in a usability lab immediately after the first part. The users were asked to perform tasks with wearable controls integrated into an apron, see Figure 8 , where three sets of controls had the same function but a different look and feel (i.e. visible buttons, ornaments, and nearly invisible buttons). The tasks were set up to resemble two different environments, namely a train setting and a home kitchen scenario.

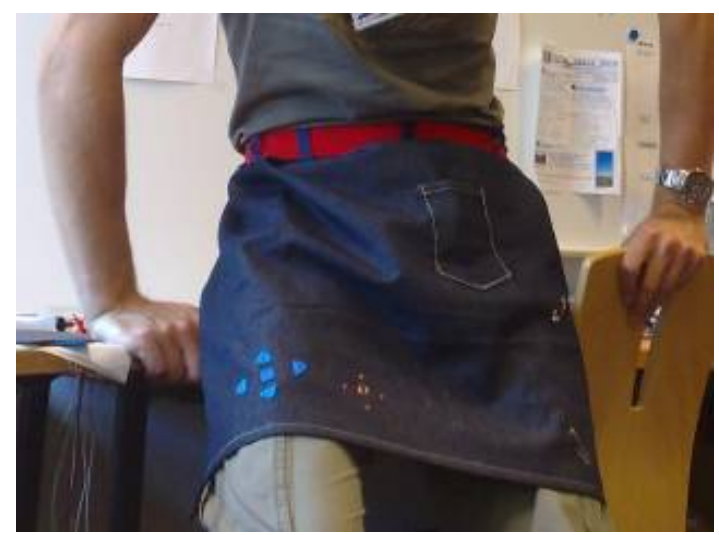

Figure 8: The apron worn by a user and adjusted such that the visible set of controls is located on the upper thigh as used in Study II.

The train setting simulated a small semi-public display integrated in the front seat of a train compartment. The user was asked to control a front seat display with apron buttons while sitting: find a 
specific channel, turn the volume up to the maximum value and down to a comfortable level. A similar task had to be executed after standing up ('in order to get coffee') and sitting down again in order to see whether a slightly moved position of the controls would have an impact. Another couple of tasks (switching to a channel and regulating the sound volume) was also performed in a standing posture with a coffee cup in hand. This was repeated with different the three wearable controls (Figure 8). The order in which the controls were used was changed with each participant.

In the kitchen scenario, home cinema devices (stereo and TV) were controlled using the controls on the apron in a similar way as described above. In this scenario, the user was standing in the kitchen, 'preparing' a meal and should control first the TV and afterwards the stereo running in the background. Here, the tasks included navigating TV channels and changing the volume. After that, the user was asked to control the music player with the same wearable controls. Here, the users were additionally asked how they would switch between devices with wearable inputs and opinions related to settings, controls and tasks were inquired.

\section{RESULTS, LESSONS LEARNED AND GUIDELINES}

In the studies we present here, the participants often had to rate a specific tool, type of interaction or give their opinion. We used an answer scheme according to a Likert scale which translates into numbers between 1 (very negative) through 3 (neutral / do not know) to 5 (very positive). For such quantitative data, we present mode values. Besides the subjective values of the participants, the observers also rated the interactions according to their observations. There was a high correlation between the ratings $(>75 \%)$, the main differences being that observers more often neglected difficulties at the first attempts of a user with a specific technique. This also indicates that users were not influenced too much by the 'presenter-bias', i.e. trying to be polite to the presenters. Citations from non-English speaking participants have been translated to English.

\subsection{Wearable Accessories}

All participants from the studies carried a mobile phone with them at that moment. Common storage places were bags and pockets suggesting that it can indeed be of additional value to them to be able to control some functionality without needing to take the phone in the hands.

The social acceptability is a large issue for the design of wearable devices and controls. As one of the initial questions, we asked where people would be prepared to use wearable controls in public. Only controls on a trouser, wrist band or separate bag received acceptable values (median values of 4 or more). Locations on the upper body like shirt or scarf were generally rejected (median values of 2 or less).

Several participants who tried the devices mentioned that because of a personal taste they do not like dangling or attachable things. Those then suggested integrating the controls into clothing like a belt or trouser. This additionally motivated us to initiate the second study described next.

\subsection{Wearable Controls}

Participants in this study could put on the apron and control the playing of media in the infrastructure as described above.
Generally speaking, the users were very positive about the applications and the ease of controlling; especially that they were not bound to a specific location in the room, that there was no line of sight to any device necessary and that there was actually no sign of any control or computing device. When asked how to switch from controlling one device to another, all but one suggested using another special button on the clothing. This indicates that when seeing how this interaction technique works, they do not think of physically walking to a device. However, if the choice of available devices grows, solutions to select the desired device like those presented in [26] are clearly superior than having one button to switch from one to the next.

\subsection{Main Guidelines}

In the course of the studies as well as during the analysis of the results, several issues have been identified that should be taken into account by application developers in this area. This section goes into some detail about the four most important guidelines that we found. Afterwards, we more briefly mention several others in a lessons-learned section.

\subsubsection{There are No Clear Expectations on Layout and Meaning}

Even for simple interfaces involving only very few controls, there seems to be no clear expectation of the arrangement, layout and meaning of the controls. For the media player control (start/pause, next, previous, volume up, volume down) for example, we had 5 different interpretations of one and the same button layout (swapping, e.g., next and previous or volume up and down). Hence one could argue this should be customizable and that idea also appealed to the participants. We implemented this version for the N800 scenario. However, an interesting observation was that all of those who expected different behavior than they found, accepted the given mapping and got used to the specific arrangement after at most 2 wrong attempts. This means that the need for configuration is may be less important if the next guideline is followed:

\subsubsection{Locating and Identifying Controls must be Quick and Easy}

For a successful design, it is essential that users can find the controls and see the functionality assigned to them visually and tactile. A tactile way of finding a control is important for all applications that should possibly be operated during other activities ("blind"). In our sample applications, for example, purely visual controls might be enough for the setting using the public display in the train seat. The other music player applications, however, are often in use while doing sports, cooking, etc. Not much surprisingly, the first reactions on the 3 control designs (visible, ornamental, invisible) were according to this statement:

"Big buttons are ugly. It would be even better if the metallic ones were part of a larger ornament. The invisible ones 'look' of course the best."

However, during the experiment, the observed and subjective grades given for the 4 tasks were, as expected, lower for the invisible controls than for the visible or ornamental ones (however, only with respect to the visible ones, the difference is statistically significant, 1 -tailed, paired t-test: $p<.03$ ). As can be 
seen in Figure 9, people found it harder to use without looking (t-tests show significant differences for all 3 button styles: $\left.\mathrm{p}_{\text {visible }}<.001, \mathrm{p}_{\text {ornamental }}<.01, \mathrm{p}_{\text {invisible }}<.0001\right)$. Nevertheless, only the invisible ones made the scores degrade in a completely unacceptable way.

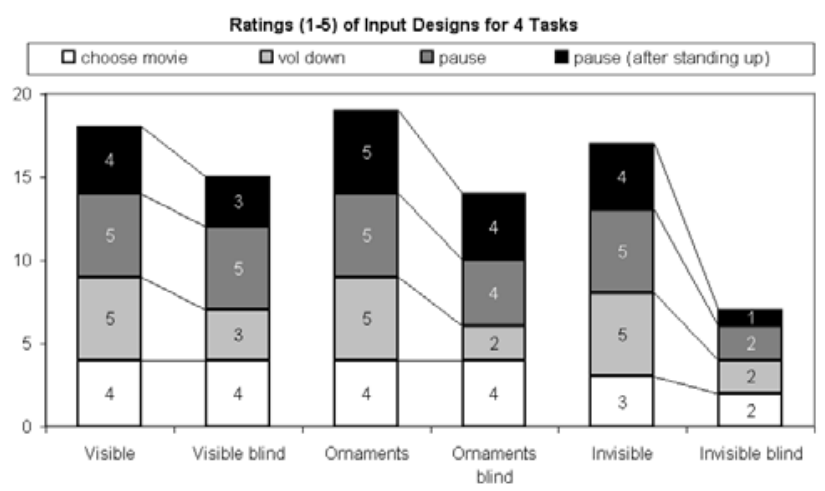

Figure 9: Subjective ratings (mode) according to a Likert scale from 1 (negative), to 3 (neutral) to 5 (positive). 4 Tasks have been evaluated with 3 different designs; each of those was also done allowing tactile search only ("blind").

This data suggests that using controls embedded in the design, are visible and tangible as well as look and feel different for different functionality is the best choice.

\subsubsection{Ensure One-handed Interaction}

Besides the appearance of controls, care must be taken to ensure that one handed interaction is possible as this is expected from a wearable control. This should not be underestimated and several existing designs can be found that use, e.g., the sleeve to embed a keypad or keyboard. This means that both arms and hands cannot serve any other purpose at the moment of input. Avoiding this is especially important for use during specific activities like cycling, motor biking, running, skating, or working. A designer can thus also decide not to follow this guideline if a thorough investigation of the usage of patterns of the target group reveals it is unnecessary. For any generic application, it is vital, though.

\subsubsection{Provide Immediate Feedback}

Even a short delay between input and action can be extremely critical. We could observe that people do not yet know or understand the way of operation of touch controls. Although all our touch control designs have a haptic impression, all people initially used those expecting some tactile, 'button-like click' feedback. Whenever there was no immediate consequence after a touch, people tried to push the electrodes harder instead of releasing and touching it again. In most controls we implemented a dwell time which required people to stay on a control for a short amount of time to tackle false sensor readings and to avoid accidentally initiated commands (see also the next section). According to observations and comments, this accounted for more than a third of the problems the users initially had in controlling the applications. The physical separation of controls and system and the several points of failure require a minimal delay between input and action (see, however, 5.4.4 about ways to avoid accidental uses). As an example of giving direct feedback even if the main effect is delayed or not directly visible, the helmet prototype could be extended with a small lamp attached to the front that provides some feedback to the user, e.g., blink when a control was touched.

\subsection{Lessons Learned}

This section briefly describes more results that we were able to draw from the studies described above.

\subsubsection{Even Minimal Enhancements Can Convince the User}

A very positive and motivating finding was that even if several scenarios arguably offered only a small increase in the ease of handling, such approaches are very much appreciated. For the designs used as remote control for a phone, more than half of the participants explicitly valued the indirect access:

"That is quite useful. No need to take phone out while [walking / running / reading / cycling / ...]. I don't see any bad sides."

Additionally, the demonstration with the augmented helmet showed that people also recognize the value of having an additional motivation to using such safety devices.

\subsubsection{Preserve the Original Functionality}

For users it is paramount that the original functionality and way of use must not be compromised. This also includes safety concerns, e.g., when embedding hardware in a bicycle helmet. It especially applies to devices that have a distinct set of functions to fulfill like the gloves.

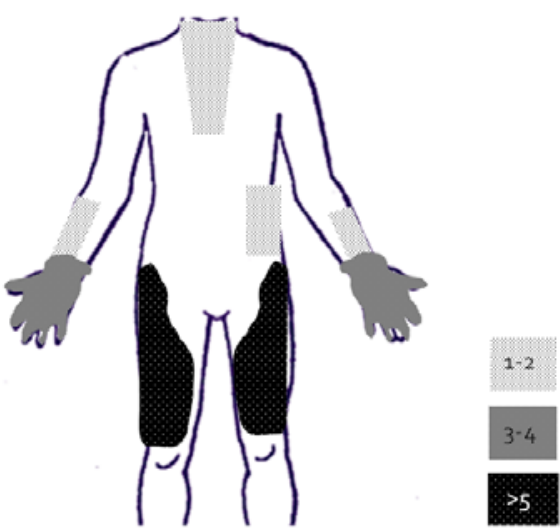

Figure 10: Preferences of people where to potentially accept wearable touch controls. The darker the color, the more often this place was indicated. The thigh was mentioned most often. This applies for standing postures only.

\subsubsection{Optimal Position of Controls Influenced by} Posture

The optimal position of controls with regard to the body shows clear trends over the whole population of participants (see Figure 10). However, body posture has an impact on optimal positions and hence the location of controls which are operated while standing may have a different slightly optimal location while sitting. Although we did not concentrate on further exploring this yet, we can say that all but one of the users raised the controls on the thighs when standing by about 6 inches. Several persons also wanted the button functions to be different while standing. We suggest using a built-in accelerometer and 
refer to, e.g., [12], [15] for projects and links to more advanced recognition methods.

It should be noted here, that all of the participants stated that they judged detachable controls to make sense. The two reasons given were that the location could be controlled and it could be used for different clothing and can be reused even if, e.g., the gloves break. However, the cost of embedding the touch electrodes (and possibly sensors) is already minimal. Only more expensive parts like the wireless connection module must be replaceable. A possible solution for the location problem could be replicating sets of controls at different positions.

\subsubsection{Need to Tackle the Fear of Accidentally Initiated Commands}

As mentioned above, we saw a timing dilemma in our experiments. On one side, users want to be sure that they don't accidentally operate a button when touching it by accident. On the other hand, longer required touch time when deliberately operating often led to frustration as there is no immediate reaction. Mechanisms that provide immediate response and also have a key lock function need to be developed. Possible solutions include using double taps (as in [25]) or operate the sensors only when the palm touches a surface slightly above the controls.

\subsubsection{More General Remarks}

For clothes as well as accessories, users put fashion forward as a main concern. This provides a good opportunity to improve the expressiveness of clothing (e.g. a technical style) but is a serious risk as people might not like the product merely because of its appearance and not because of its function or functionality. Including fashion and clothing designers for a commercial project is therefore mandatory.

Besides fashion design, one of the issues we confirmed in the interviews is that there exists a variety of specific interface needs, in particular with regards to the gloves (e.g. for different work environments).

Additionally, an often expressed issue is that people do not want to be concerned with another device to charge. Long battery life and ease of handling, storing and charging is critical. We propose to employ a simple plug mechanism to connect and draw power from the phone, e.g., implemented in the phone bags or a pocket (we saw that more than $60 \%$ of the participants kept it in a trouser pocket). We refer to Toney et al. [29] for a way of simplifying the management (which includes recharging) of smart clothing with enhanced clothes hangers.

Generally speaking, integration of controls into garment enables possibilities for interactions during activities like working or cycling that were difficult or not possible. This shows the utility of wearable controls but at the same time introduces new risks to these activities.

\section{CONCLUSION}

We presented several prototypes of wearable accessories and clothing that feature different types of touch sensitive controls. The technology of capacitive sensing that we used was briefly introduced. A focus of the work has been to create and use tools to quickly generate and evaluate objects and applications using touch controls. We have provided references that show how these techniques can be implemented in a larger scale at production level. The working prototypes have been evaluated in two user studies and guidelines have been extracted from the results. For several of the issues critical to the success of wearable controls that were identified, solutions have been suggested. We extracted four main aspects that we found to be important for projects using such approaches and technologies. First, it is a very tough problem to find a design and layout of button inputs that match the ideas of a majority of users. This issue can be softened when having a clear mapping from appearance to function. This leads to the second recommendation that finding a specific control must be made as simple and quick as possible. This requires well designed visible as well as haptic cues. We also provide hints as to the location on the body that is preferred by people. Thirdly we stress that it is most important to have the controls placed in a way that one-handed interaction is easily possible. The fourth main guideline is that the system needs to ensure that feedback for the actions needs to be without delays; otherwise users can not be sure about whether or not their input had been registered which then can lead to frustration or accidental multiple button presses. We believe that these guidelines and the issues that we encountered can be valuable for creating touch input on clothes.

\subsection{Future Work}

On one part of the apron we reserved an area which we will use to learn about the different ideas of people about their personal optimized layout of specific controls. Additionally, we plan to work with professional designers to create different designs of basic sets of controls. For that, we built a set of button-like wires that can be overlaid on designs and connected to the sensor platform within a few minutes and therefore tested on the fly (see Figure 11)
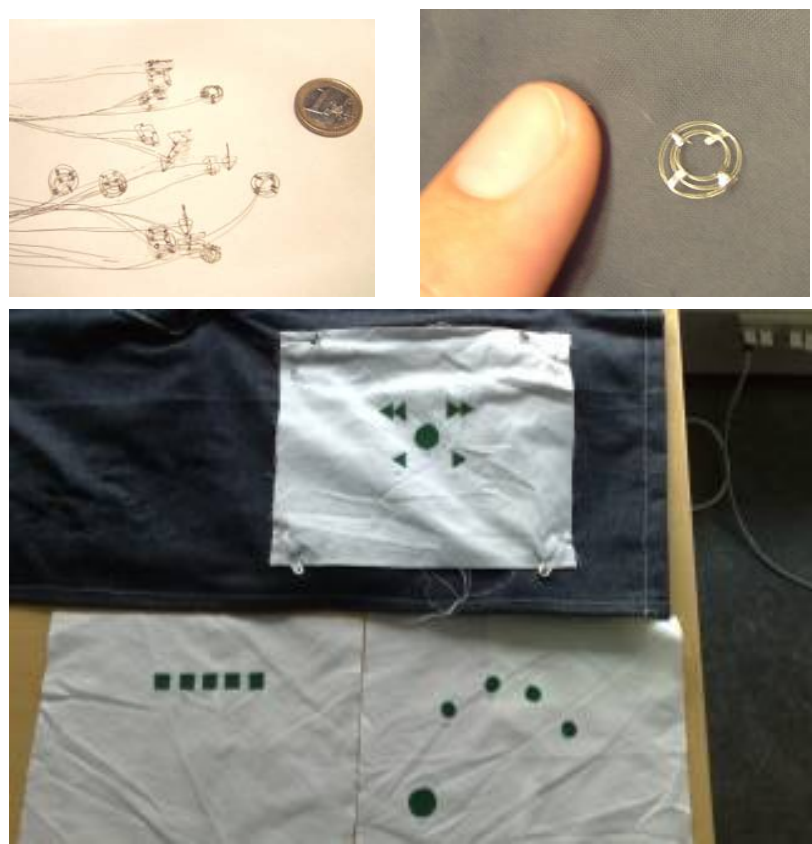

Figure 11: Prototyping material for quickly testing control layouts. Several strips of conductive wires with ornamental buttons (top) and several easily exchangeable layouts for the media player control (bottom). 


\section{REFERENCES}

[1] Buechley, L. A Construction Kit for Electronic Textiles. In Proc. ISWC'06. 28-32. 2006

[2] Gemperle, F., Kasabach, C., Stivoric, J., Bauer, M., Martin, R. Design for Wearability. In Proc. ISWC'98. 116. 1998

[3] Hartmann, B., Klemmer, S.R., Bernstein, M., Abdulla, L., Burr, B., Robinson-Mosher, A., Gee, J., Reflective Physical Prototyping through Integrated Design, Test, and Analysis. In Proc. UIST'06. 299-308. 2006

[4] Hannikainen, J., Mikkonen, J., Vanhala, J. Button Component Encasing for Wearable Technology Applications. In Proc. ISWC'05. 204-205. 2005

[5] Hurford, R., Martin, A., Larsen, P., Designing Wearables. In Proc. ISWC'06. 133-134. 2006

[6] Jung, S., Lauterbach, C., Weber, W. A Digital Music Player Tailored for Smart Textiles: First Results. Avantex Symposium. 2002

[7] Knight, J. F., Baber, C., A Tool to Assess the Comfort of Wearable Computers. Human Factors. 47(1). 77-91. 2005

[8] Knight, J.F., Deen-Williams, D., Arvanitis, T.N., Baber, C., Sotiriou, S., Anastopoulou, S., Gargalakos, M., Assessing the Wearability of Wearable Computers. In Proc. ISWC'06. 7582. 2006

[9] Lee, S., Buxton, W., Smith, K. C. A Multi-Touch Three Dimensional Touch-Sensitive Tablet. In Proc. CHI'85. 21 25. 1985

[10] Linz, T., Kallmayer, C., Aschenbrenner, R., Reichl, H. Embroidering Electrical Interconnects with Conductive Yarn for the Integration of Flexible Electronic Modules into Fabric. In Proc. ISWC'05. 86-91. 2005

[11] Liu, Y., Liu, X., Jia, Y. Hand-Gesture Based Text Input for Wearable Computers. In Proc. ICVS'06. 8. 2006

[12] Lombriser, C., Bharatula, N.B., Roggen, D., Tröster, G. OnBody Activity Recognition in a Dynamic Sensor Network. In Proc. BodyNets'07. 2007

[13] Lyons, K., Plaisted, D., Starner, T. Expert Chording Text Entry on the Twiddler One-Handed Keyboard. In Proc. ISWC'04. 94-101. 2004

[14] Marculescu, D., Marculescu, R., Zamora, N.H., StanleyMarbell, P., Khosla, P.K., Park, S., Jayaraman, S., Jung, S. Lauterbach, C., Weber, W., Kirstein, T., Cottet, D., Grzyb, J., Tröster, G., Jones, M., Martin, T., Nakad, Z. Electronic Textiles: A Platform for Pervasive Computing. Proceedings of the IEEE. 91(12). 2003

[15] Mattmann, C., Tröster, G. Design Concept of Clothing Recognizing Back Postures. In Proc. ISSS-MDBS'06. 2006

[16] Mayol, W.W., Tordoff, B., Murray, D.W. On the Positioning of Wearable Optical Devices. Technical Report OUEL224101. Oxford University. 2001

[17] McCann, J., Hurford, R., Martin, A. A Design Process for the Development of Innovative Smart Clothing that
Addresses End-User Needs from Technical, Functional, Aesthetic and Cultural View Points. In Proc. ISWC'05. 7077. 2005

[18] Orth, M., Post, R., Cooper, E. Fabric computing Interfaces. In Proc. CHI'98. 331-332. 1998

[19] Park, S., Mackenzie, K., Jayaraman, S. The Wearable Motherboard: a Framework for PMIP. In Proc. DAC'02. 170-174. 2002

[20] Post, E. R., Orth, M., Russo, P. R., Gershenfeld, N. Ebroidery: Design and Fabrication of Textile-Based Computing. IBM Systems Journal. 39(3-4). 840-860. 2000

[21] Power, E.J. T. Dias. Knitting of Electroconductive Yarns. In Proc. IEE Eurowearable '03. 55-60. 2003.

[22] Randell, C., Wearable Computing: A Review. Technical Report CSTR-06-004. University of Bristol. 2005

[23] Rantanen, J., Karinsalo, T., Mäkinen, M., Talvenmaa, P., Tasanen, M., Vanhala, J., Alfthan, N., Impiö, J., Malmivaara, M., Matala, R., Reho, A. Smart Clothing for the Arctic Environment. In Proc. ISWC'00. 15. 2000

[24] Rhodes, B., Mase, K. Wearables in 2005. IEEE Pervasive Computing. 5(1). 92. 2006

[25] Ronkainen, S., Häkkilä, J., Kaleva, S., Colley, A., Linjama, J. Tap Input as an Embedded Interaction Method for Mobile Devices. In Proc. TEI’07. 263-270. 2007

[26] Rukzio, E., Leichtenstern, K., Callaghan, V., Holleis, P., Schmidt, A., Shiaw-Yuan Chin, J. An Experimental Comparison of Physical Mobile Interaction Techniques: Touching, Pointing and Scanning. In Proc. Ubicomp'06, 87104. 2006

[27] Thomas, B. H. Minimal Social Weight User Interactions for Wearable Computers in Business Suits. In Proc. ISWC'07. 57. 2002

[28] Thomas, B., Grimmer, K., Makovec, D., Zucco, J., Gunther, B. Determination of Placement of a Body-Attached Mouse as a Pointing Input Device for Wearable Computers. In Proc. ISWC'99. 193. 1999

[29] Toney, A., Thomas, B. H., Marais, W. Managing Smart Garments. In Proc. ISWC'06. 91-94. 2006

[30] Wade, E., Asada, H. Conductive Fabric Garment for a CableFree Body Area Network. IEEE Pervasive Computing. 6(1). 52-58. 2007

[31] Wade, E., Asada, H. Wearable DC Powerline Communication Network Using Conductive Fabrics, In Proc. ICRA'04. 4085-4090. 2004

[32] Weiser, M. The Computer for the Twenty-First Century. Scientific American. 94-10. 1991

[33] Witt, H., Nicolai, T., Kenn, H. The WUI-Toolkit: A ModelDriven UI Development Framework for Wearable User Interfaces. In Proc. ICDCSW'07. 43 